\title{
Channel Measurements and Models for High-Speed Train Communication Systems: A Survey
}

\author{
Cheng-Xiang Wang, Senior Member, IEEE, Ammar Ghazal, Student Member, IEEE, Bo Ai, Senior Member, IEEE,
} Yu Liu, and Pingzhi Fan, Fellow, IEEE

\begin{abstract}
The recent development of high-speed trains (HSTs), as an emerging high mobility transportation system, and the growing demands of broadband services for HST users introduce new challenges to wireless communication systems for HSTs. Accurate and efficient channel models considering both largescale and non-stationary small-scale fading characteristics are crucial for the design, performance evaluation, and parameter optimization of HST wireless communication systems. However, the characteristics of the underlying HST channels have not yet been sufficiently investigated. This article first provides a comprehensive review of the measurement campaigns conducted in different HST scenarios and then addresses the recent advances in HST channel models. Finally, key challenges of HST channel measurements and models are discussed and several research directions in this area are outlined.
\end{abstract}

Index Terms-High-speed train channels, channel measurements, non-stationary channel models, statistical properties.

\section{INTRODUCTION}

High-mobility scenarios, e.g., high-speed train (HST) and vehicle-to-vehicle (V2V) scenarios, are expected to be typical scenarios for fifth generation $(5 \mathrm{G})$ communication systems [1]. Unlike V2V communication channels that have been thoroughly investigated in the literature [2], [3], a comprehensive study of HST communication channels is still missing. With the rapid development of HSTs, an increasing volume of wireless communication data is required to be transferred to train passengers. HST users demand high network capacity and reliable communication services regardless of their locations or speeds. To satisfy these demands, HST wireless communication systems have to overcome many challenges resulting from the high speed of the train that can easily exceed $250 \mathrm{~km} / \mathrm{h}$, such as fast handover, fast travel through widely

The authors would like to acknowledge the support from the EU FP7 QUICK project (Grant no. PIRSES-GA-2013-612652), EU H2020 5G Wireless project (Grant no. 641985), National 863 project in 5G by Ministry of Science and Technology in China (Grant no. 2014AA01A706), the National Natural Science Foundation of China (Grant no. 61222105), the Natural Science Base Research Plan in Shaanxi Province of China (Grant no. 2015JM6320), National 973 Program (Grant no.2012CB316100), and 111 project (Grant no.111-2-14).

C.-X. Wang (corresponding author) and A. Ghazal are with the Institute of Sensors, Signals and System, School of Engineering and Physical Sciences, Heriot-Watt University, Edinburgh, EH14 4AS, UK (e-mail: \{chengxiang.wang, ag289\}@hw.ac.uk).

B. $\mathrm{Ai}$ is with the State Key Laboratory of Rail Traffic Control and Safety, Beijing Jiaotong University, Beijing 100044, China (e-mail: boai@bjtu.edu.cn).

Y. Liu is with the R\&D Center on B4G Wireless Communication Networks, School of Information Science and Engineering, Shandong University, Shandong 250100, China (email: xinwenliuyu@163.com).

P. Fan is with the Institute of Mobile Communications, Southwest Jiaotong University, Chengdu, Sichuan 610031, China (e-mail: p.fan@ieee.org). diverse scenarios, and large Doppler spreads [4], [5] besides some challenges inherited from conventional trains such as high penetration losses, limited visibility in tunnels, and the harsh electromagnetic environment [6].

Since 1998, the Global System for Mobile Communication Railway (GSM-R) has widely been adopted as Europe standard for train communications and control. However, GSM-R can only provide a data rate of up to $200 \mathrm{kbps}$ [7], besides the fact that it is mainly used for train control rather than providing communications for train passengers [8]. Therefore, GSM-R cannot meet the requirements for future high speed data transmissions [9] and International Union of Railways has recommended that GSM-R has to be replaced by longterm evolution-Railway (LTE-R) [10]-[16], which is a broadband railway wireless communication system based on LTEAdvanced (LTE-A) [17]. Nevertheless, both systems still adopt the conventional cellular architecture where mobile stations (MSs) inside trains communicate directly with outdoor base stations (BSs). Such an architecture leads to a spotty coverage and high penetration losses of wireless signals traveling through the metal carriages of HSTs. In addition, the receiving signals at MSs on board will experience fast changing channels resulting in high signaling overhead and high possibility of drop calls and handover failure [18].

The aforementioned problems can be mitigated by deploying other cellular architectures, such as distributed antenna system (DAS) [19]-[21], coordinated multipoint (CoMP) [22], [23], mobile relay station (MRS) [24]-[27] (or mobile femtocell [1], [28], [29]) technologies, or a combination of these architectures, e.g., DAS with MRS [30] or CoMP with MRS [31]. In a DAS, distributed antenna elements are connected to a BS via wires or fibers (radio over fibers $(\mathrm{RoF})$ ) [32], [33] to provide considerable gain in coverage and capacity in comparison with the conventional cellular architecture. The spatially separated antenna elements can be used to transmit the same signal at different locations to provide spatial diversity against the fading. Combined with spatial diversity, frequency reuse in the DAS is an effective technique to increase system capacity. The enhancement in spectral efficiency of DASs in comparison with conventional systems was presented in [19]. In [20], the authors analyzed the deployment of DAS over HST communication systems and some of the resulting problems such as the coverage of the remote antenna units (RAUs) and echo channel effect. In CoMP systems, the transmission of neighboring BSs will be coordinated in the downlink while the received signals at the uplink will be jointly processed. This will reduce the inter-cell interference 
and improve the cell edge throughput. CoMP systems will also provide an enhanced channel capacity by using the statistically independent properties of the channels resulting from the wide spatial separation of antenna elements. Adopting mobile femtocell architecture in HST communication systems can be performed by deploying dedicated MRSs on the surface of the train to extend the coverage of the outdoor BS into train carriages. As a result, we will have two channels: an outdoor channel between the BS and MRS, and an indoor one between the MRS and an MS of a train passenger as illustrated in Fig. 1. In this case, the BS will mainly communicate with the MRS at high data rates instead of communicating with large numbers of MSs directly. An MRS and its associated MSs within a train carriage are all viewed as a single unit to the BS, while the MSs will see the relevant MRS as a regular BS. It follows that an MRS can perform a group handover on behalf of all its associated MSs, which can greatly reduce the frequent handover burden of the HST system [29]. Since the complexity of radio resource allocation (i.e., transmit power, data rates, scheduling, power and frequency allocation, and antenna selection) in a BS is related to the number of active users [19], the radio resource management complexity in one BS will be reduced significantly when dealing with a "group of users" rather than individuals. This promising MRS technology has been adopted by IMT-Advanced (IMT-A) [34] and WINNER II [35].

Moreover, the transmitter (Tx) and receiver $(\mathrm{Rx})$ of a HST wireless communication system encounter different channel conditions due to the difference of surrounding geographical environments. The HST environment can be generally classified into the following main scenarios: open space, viaduct, cutting, hilly terrain, tunnels, and stations. Considering some unique setup of the aforementioned scenarios and some other special HST scenarios, HST environment can be further classified into 12 scenarios [37]. HSTs can operate across one or more of these scenarios during its travel. The propagation characteristics change significantly with the change of environments and the distance between the Tx and Rx, even in the same terrain. Scenarios have close relationship with channel modeling and measurements. Most standard channel models in the literature, like UMTS [38], COST 2100 [39], and IMT-2000 [40], failed to introduce any of the HST scenarios. The moving networks scenario in the WINNER II channel model [35] and rural macro-cell (RMa) scenario in the IMT-A channel model [34] have only considered a rural environment for HSTs, while neglecting other HST scenarios. The aforementioned propagation scenarios will be introduced and explained in detail in Section II.

The features of HST channels, e.g., non-stationarity and large Doppler shift, significantly differ from those of lowmobility mobile cellular communication channels. Therefore, many measurement campaigns have been conducted in the literature to understand the underlying physical phenomenon in HST propagation environments. Accurate channel models that are able to mimic key characteristics of wireless channels play an important role in designing and testing HST communication systems. Realistic and reliable large-scale fading channel models, i.e., path loss (PL) and shadowing fading

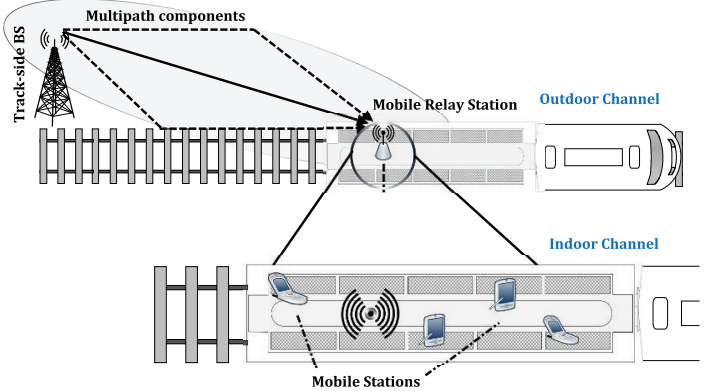

Fig. 1. A HST communication system deploying MRSs.

(SF) models, are indispensable for efficient and trustworthy network deployment and optimization. Small-scale fading channel models are crucial in physical layer design in order to develop and test different schemes, such as diversity of transmission/reception, error correction coding, interleaving, and equalization algorithms. Inaccurate channel models may lead to over-optimistic or over-pessimistic performance evaluation results that will result in misjudgments in product development. Moreover, inaccurate channel models may lead to inaccurate link budgets that will result in huge errors of the estimated maximum distance between adjacent BSs. Consequently, this will cause poor coverage and increased drop calls due to failed handovers between BSs when the distance is underestimated, and unnecessary overlapped coverage area with unjustified installation and maintenance cost of the extra installed BSs when the distance is overestimated [36]. In the literature, several large-scale and small-scale fading HST channel models were proposed. This article will focus on the recent advances in HST channel measurements and modeling and their future challenges.

The rest of this paper is organized as follows. In Section II, an overview of HST channel measurements is provided. The state-of-the-art of HST channel models is presented in Section III. Future research directions in HST channel measurements and models are outlined in Section IV. Finally, concluding remarks are highlighted in Section V.

\section{HST ChannEl MEASUREMEnts}

Special attention has been given to HST channel measurements in recent years. Due to the high speed of the train and the hostile HST environments, conducting accurate channel measurements for HST communication systems is challenging and needs to address particular hardware and software requirements, e.g., robustness, scalability, hardware redundancy and traceability [41]. Many measurement campaigns [42], [44]-[48], [51]-[75], [77]-[79], [81]-[90] for different HST environments were presented in the literature. Here, we will briefly review and classify the important measurements for HST communications according to the scenarios, cellular architecture, measurements' setup parameters (i.e., antenna configuration, carrier frequency, and bandwidth), and measured channel statistics, as shown in Table I. 
TABLE I

IMPORTANT HST CHANNEL MEASUREMENTS.

\begin{tabular}{|c|c|c|c|c|c|c|c|}
\hline Ref. & Cellular Architecture & Scenario & Carrier Frequency & Bandwidth & Antenna & Train Speed & Channel Statistics \\
\hline [42] & MRS & N/A & $2.2 \mathrm{GHz} / 5.2 \mathrm{GHz}$ & $20 \mathrm{MHz}$ & SISO & $270 \mathrm{~km} / \mathrm{h}$ & PL \\
\hline [44] & MRS & N/A & $2.35 \mathrm{GHz}$ & $100 \mathrm{MHz}$ & SISO & N/A & PL, DS, $K$ \\
\hline [45] & Conventional & Open space & $2.6 \mathrm{GHz}$ & $20 \mathrm{MHz}$ & SISO & $370 \mathrm{~km} / \mathrm{h}$ & PL, DS, DF, PDP \\
\hline [46] & Conventional & Open space & $2.5 \mathrm{GHz}$ & $50 \mathrm{MHz}$ & MISO/SIMO & $290 \mathrm{~km} / \mathrm{h}$ & DS, AoA, AoD, PAS, DF \\
\hline [47] & Conventional & Open space & $5.2 \mathrm{GHz}$ & $120 \mathrm{MHz}$ & SIMO & $350 \mathrm{~km} / \mathrm{h}$ & PL, SF, $K$, DS, PDP, AS \\
\hline [48] & MRS & Open space & $930 \mathrm{MHz}$ & $200 \mathrm{kHz}$ & SISO & $350 \mathrm{~km} / \mathrm{h}$ & PL, SF \\
\hline [51] & MRS & Viaduct & $930 \mathrm{MHz}$ & $200 \mathrm{kHz}$ & SISO & $340 \mathrm{~km} / \mathrm{h}$ & PL, $K$ \\
\hline [52] & MRS & Viaduct & $930 \mathrm{MHz}$ & $200 \mathrm{kHz}$ & SISO & N/A & PL \\
\hline$[53],[54]$ & MRS & Viaduct & $930 \mathrm{MHz}$ & $200 \mathrm{kHz}$ & SISO & $300 \mathrm{~km} / \mathrm{h}$ & $K$ \\
\hline$[55]$ & MRS & Viaduct & $930 \mathrm{MHz}$ & $200 \mathrm{kHz}$ & SISO & $350 \mathrm{~km} / \mathrm{h}$ & PL, SF \\
\hline$[56]-[58]$ & MRS & Viaduct & $930 \mathrm{MHz}$ & $200 \mathrm{kHz}$ & SISO & $350 \mathrm{~km} / \mathrm{h}$ & PL \\
\hline [59], [60] & MRS & Viaduct & $930 \mathrm{MHz}$ & $200 \mathrm{kHz}$ & SISO & $350 \mathrm{~km} / \mathrm{h}$ & SF \\
\hline [61] & MRS & Viaduct & $930 \mathrm{MHz}$ & $200 \mathrm{kHz}$ & SISO & $300 \mathrm{~km} / \mathrm{h}$ & PDF, LCR, AFD, CDF, FM \\
\hline [62] & MRS & Viaduct & $2.35 \mathrm{GHz}$ & $10 \mathrm{MHz}$ & SISO & $240 \mathrm{~km} / \mathrm{h}$ & PL, DS, $K$ \\
\hline$[63]$ & MRS & Viaduct & $2.35 \mathrm{GHz}$ & $50 \mathrm{MHz}$ & SISO & $196 \mathrm{~km} / \mathrm{h}$ & $\mathrm{DS}, K, \mathrm{SF}$ \\
\hline$[64],[65]$ & MRS & Viaduct & $930 \mathrm{MHz}$ & $200 \mathrm{kHz}$ & SISO & $360 \mathrm{~km} / \mathrm{h}$ & $\mathrm{PL}, K, \mathrm{SF}, \mathrm{FD}, \mathrm{LCR}, \mathrm{AFD}$ \\
\hline$[66]$ & MRS & Viaduct & $2.6 \mathrm{GHz}$ & $20 \mathrm{MHz}$ & SISO & $370 \mathrm{~km} / \mathrm{h}$ & PL, SF, DS, $K$ \\
\hline$[67],[68]$ & MRS & Viaduct & $2.35 \mathrm{GHz}$ & $50 \mathrm{MHz}$ & SISO & $200 \mathrm{~km} / \mathrm{h}$ & PSD, DF, AoA, $K$ \\
\hline [69] & MRS & Cutting & $930 \mathrm{MHz}$ & $200 \mathrm{kHz}$ & SISO & $320 \mathrm{~km} / \mathrm{h}$ & PL, SF \\
\hline [70] & MRS & Cutting & $930 \mathrm{MHz}$ & $200 \mathrm{kHz}$ & SISO & $350 \mathrm{~km} / \mathrm{h}$ & PL, $K$ \\
\hline [71] & MRS & Cutting & $930 \mathrm{MHz}$ & $200 \mathrm{kHz}$ & SISO & $295 \mathrm{~km} / \mathrm{h}$ & $K, \mathrm{FD}$ \\
\hline [72] & MRS & Cutting & $930 \mathrm{MHz}$ & $200 \mathrm{kHz}$ & SISO & $350 \mathrm{~km} / \mathrm{h}$ & $\mathrm{PL}, K, \mathrm{SF}, \mathrm{FD}, \mathrm{LCR}, \mathrm{AFD}$ \\
\hline [73] & MRS & Cutting & $2.35 \mathrm{GHz}$ & $50 \mathrm{MHz}$ & SISO & $200 \mathrm{~km} / \mathrm{h}$ & $\mathrm{PL}, K, \mathrm{SF}, \mathrm{DF}$ \\
\hline$[74]$ & MRS & Cutting & $2.35 \mathrm{GHz}$ & $50 \mathrm{MHz}$ & SISO & $200 \mathrm{~km} / \mathrm{h}$ & $\mathrm{DS}, \mathrm{DF}$ \\
\hline$[75],[77]$ & Conventional & Hilly Terrain & $2.4 \mathrm{GHz}$ & $40 \mathrm{MHz}$ & SISO & $295 \mathrm{~km} / \mathrm{h}$ & PL, SF, $K$ \\
\hline$[78]$ & Conventional & Tunnel & $2.154 \mathrm{GHz}$ & $30 \mathrm{MHz}$ & SISO & N/A & PL, DS \\
\hline [79] & MRS\& DAS & Tunnel & $930 \mathrm{MHz}$ & $200 \mathrm{kHz}$ & SISO & N/A & PL \\
\hline [81] & MRS & Station & $930 \mathrm{MHz}$ & $200 \mathrm{kHz}$ & SISO & N/A & PL \\
\hline$[82]$ & MRS & Station & $930 \mathrm{MHz}$ & $200 \mathrm{kHz}$ & SISO & N/A & $\mathrm{PL}, K, \mathrm{SF}, \mathrm{FD}, \mathrm{LCR}, \mathrm{AFD}$ \\
\hline$[85]$ & MRS & Various & $930 \mathrm{MHz}$ & $200 \mathrm{kHz}$ & SISO & $350 \mathrm{~km} / \mathrm{h}$ & PL, PDF, DS, PDP \\
\hline$[86]$ & MRS & Various & $930 \mathrm{MHz}$ & $200 \mathrm{kHz}$ & SISO & $340 \mathrm{~km} / \mathrm{h}$ & SI \\
\hline [87], [88] & MRS & Various & $930 \mathrm{MHz}$ & $200 \mathrm{kHz}$ & SISO & $340 \mathrm{~km} / \mathrm{h}$ & SF \\
\hline$[83]$ & MRS & Various & $930 \mathrm{MHz}$ & $200 \mathrm{kHz}$ & SISO & $290 \mathrm{~km} / \mathrm{h}$ & $K$, LCR, AFD \\
\hline [89], [90] & MRS & Various & $2.1 \mathrm{GHz}$ & $3.84 \mathrm{MHz}$ & SISO & $240 \mathrm{~km} / \mathrm{h}$ & $\mathrm{PL}, K, \mathrm{DS}, \mathrm{PDP}$ \\
\hline [91] & Conventional & Various & $2.1 \mathrm{GHz}$ & $3.84 \mathrm{MHz}$ & SISO & $300 \mathrm{~km} / \mathrm{h}$ & PL, PDP \\
\hline \multicolumn{8}{|c|}{$\begin{array}{l}\text { SISO: single-input single-output; MISO: multiple-input single-output; SIMO: single-input multiple output; PL: path loss; DS: RMS delay spread; } K \text { : Ricean } \\
K \text {-factor; PDP: power delay profile; AS: angular spread; AoA: angles of arrival; AoD: angles of departure; PAS: power azimuth spectrum; DF: Doppler } \\
\text { frequency; SF: shadow fading; FD: fade depth; LCR: level crossing rate; AFD: average fade duration; PDF: Probability density function; CDF: cumulative } \\
\text { distribution function; FM: fading margin; PSD: power spectrum density, SI: stationarity interval }\end{array}$} \\
\hline
\end{tabular}

\section{A. HST Propagation Scenarios}

HST environments can be roughly classified into the following 6 scenarios: open space, viaduct, cutting, hilly terrain, tunnels and stations.

1) In the open space scenario [46], also called plain scenario [45], the $\mathrm{Rx}$ is moving at a very high speed in a rural area where the BS antenna is much higher than the surroundings [48]. This environment focuses on large cells and continuous coverage where the link between the fixed Tx and moving Rx normally has a dominant line-of-sight ( $\mathrm{LOS}$ ) component. However, after a certain distance, called breakpoint distance, the impact of the sparse scatterers will be noticed at the $\mathrm{Rx}$ represented by non-LoS (NLoS) components. As a result, the slopes of the PL and Ricean $K$-factor will be noticeably changed at the breakpoint leading to dual-slope PL model [49]. It has been proved that there is a strong link between the breakpoint distance and the antenna height. For a certain site, as the antenna height decreases, the breakpoint moves closer to the Tx. This is because a bigger Fresnel zone is intercepted by the ground, usually covered by vegetation, when the antenna height is lower. Furthermore, due to the influences of different environments, slight variations in the breakpoint

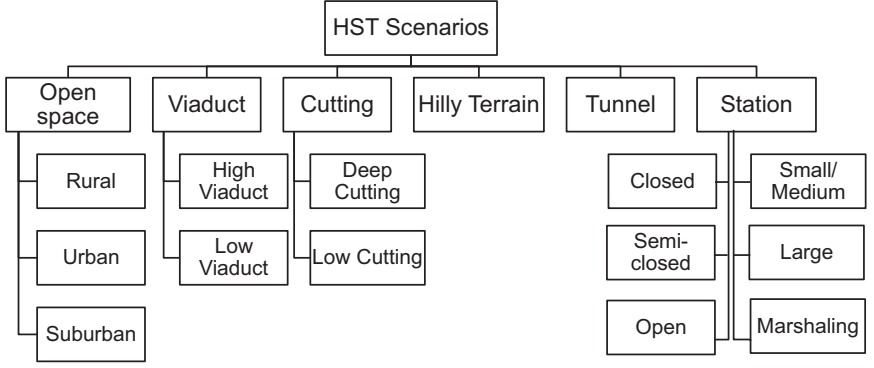

Fig. 2. Classification of HST Scenarios.

distance can be noticed in different scenarios. Therefore, it can be concluded that the breakpoint distance is mainly determined by the antenna height with slight environmental effects [50]. Based on the geographic nature and the distribution/height of the surrounding scatterers, the open scenarios can be further classified into rural [47], urban, and suburban as illustrated in Fig. 2.

2) The viaduct scenario is common for HSTs as they often operate on viaducts [51]-[67]. The main purpose of viaducts is to ensure the smoothness of the rail and the high speed 
of the train. In this scenario, the radio reflection, scattering, and diffraction caused by nearby scatterers, e.g., trees and buildings, can also be reduced significantly. The viaduct height and relative BS height have great influence on the received signal. Because of the relatively high altitude of the viaduct in comparison with the surrounding terrain, the LoS component is dominant in this scenario. However, the sparsity of the scatterers in the environment around the viaduct will still influence the received signal at the $\mathrm{Rx}$ [55]. Based on the relative altitude between the scatterers and the viaduct, this scenario can be further classified into high viaduct and low viaduct scenarios. In the former, most scatterers located within $50 \mathrm{~m}$ from the viaduct are lower than the surface of the viaduct and therefore their impact on the propagation characteristics is negligible. In the low viaduct scenario [67], [68], some of the nearby scatterers are higher than the surface of the viaduct and consequently they introduce rich reflections and scattering components that may result in a severe shadow fading and/or extra pathloss [37].

3) The cutting scenario is another common scenario for HST wireless communications [69]-[74]. It represents an environment where the HST passes a U-shaped geographical cut surface between the hills. The cutting is widely used for HST construction to ensure the smoothness of the rail and help to achieve a high speed of the train when passing through hills. The propagation of radio waveforms in this scenario is significantly affected by the steep walls on both sides. The LoS component can be observed along the route of the HST in this scenario. Here, we can recognize between two cutting scenarios: deep cutting if the receive antenna mounted on top of the train is lower than the upper eave of the cutting and low cutting if the height of the upper eave is lower than the top of the receive antenna.

4) In the hilly terrain scenario [75], [77], the surrounding environment is densely scattered with objects distributed irregularly and non-uniformly. With high-altitude transmit antennas and low-attitude obstacles, the LoS component is observable and can be detected along the entire railway. However, multipath components scattered/reflected from the surrounding obstacles will cause serious constructive or destructive effects on the received signal and therefore influence the channel's fading characteristics.

5) The tunnel scenario represents an environment where HST passes through tunnels [78], [79] with different lengths ranging from hundreds of meters to several kilometers. The length, size, and shape of the tunnels and the encountered waveguide phenomena have significant impact on the communication channel. Because of the long limited space, bounding of tunnel, and poor smoothness of the interior wall, propagation characteristics of signals in tunnels are quite different from other scenarios. To overcome the problem of the limited visibility encountered in tunnels and to design an optimal wireless communication network, leaky feeders and DAS were often deployed. However, as HST may require long tunnels, leaky feeder solution are more expensive especially at high operating frequencies and its maintenance is considerable complex [93]. As a result, DAS is more practical [94]. It can provide considerable gain in coverage and capacity, and provide spatial diversity against the fading by using the antenna elements at different locations. It also has advantages in future applications such as higher distance between repeaters, and easy maintenance after being opened, which can meet the continuous and high quality wireless communication of users in the train inside the tunnel.

6) The stations scenario represents the railway facility where HSTs stop regularly to load/unload passengers stations [81], [82]. HST stations can be classified according to their size or architecture. Based on the size of the station, which reflects the estimated communication traffic, station scenario can be categorized into small to medium size stations, large stations, and marshalling stations [37]. From the architecture perspective, that impacts the propagation characteristics inside the station, three HST station scenarios can be recognized, i.e., open station, semi-closed station, and closed station [82] as illustrated in Fig. 2. Table II briefly summarizes the description and the key-parameters of the aforementioned scenarios.

The aforementioned scenarios are the most encountered ones in HST environment. However, recent measurement campaigns have shed some light on other special HST scenarios such as crossing bridges [84]. Besides the previous "individual" scenarios, HST may encounter more than one scenario in one wireless communication cell in what so called combination scenarios [37]. Two combination scenarios are reported in the literature. The first one is a combination between tunnel and viaduct where viaducts are usually used as transition between tunnels in mountain environment. The frequent transition between tunnel and viaduct will increase the severity of fading at the transition points causing a drop in the communication quality. The second combination is between cutting scenarios, i.e., deep and low cuttings, and rural scenario. The frequent and fast transition between these scenarios can degrade the quality of the communication link and makes signal prediction quite challenging.

\section{B. Channel Statistics}

Channel statistics are essential for the analysis and design of a communication system. Most of HST measurement campaigns have concentrated on large-scale fading statistics, like path loss (PL) and shadowing. The measurement campaign, presented in [42], studied the PL in HST channels when the Tx and Rx were located inside the same HST carriage and when they were located in different carriages. The measured results showed that the waves traveling inside the train carriages are dominant compared to the ones reflected from scatterers outside the HST due to the high penetration loss of wireless signals traveling through the metal body of the carriages. On the contrary, the waves reflected from outer scatterers are dominant compared to the waves traveling inside the train carriages when the communicated devices are located in different carriages due to the high insulation between these carriages. In [44], the PL of indoor wideband HST channels was also investigated using two different indoor Tx configurations, i.e., omni-directional antenna mounted on the ceiling of the HST and a planner antenna mounted on the wall of the carriage. Measurements showed that the channel between the Tx planner 
TABLE II

HST SCENARIOS

\begin{tabular}{|c|c|c|c|}
\hline Scenario & Description & LoS/NLoS & Key Parameters \\
\hline Open Space & Refers to rural, urban, and suburban areas & LoS & Density/height of surrounding scatterers \\
\hline Viaduct & $\begin{array}{l}\text { A bridge that ensures the smoothness of the rail } \\
\text { High speeds of the train can be achieved } \\
\text { Radio reflection, scattering, and diffraction can be reduced } \\
\text { Can be classified into high and low viaducts }\end{array}$ & $\mathrm{LoS}$ & $\begin{array}{l}\text { Height of the viaduct } \\
\text { BS' height relative to the viaduct's height } \\
\text { Height of surrounding scatetterers }\end{array}$ \\
\hline Cutting & $\begin{array}{l}\text { A U-shaped geographical cut that ensures the smoothness of the rail } \\
\text { The propagation of radio waveforms is significantly affected by the } \\
\text { steep walls and the vegetation on both sides of the cutting } \\
\text { Can be classified into deep and low cuttings }\end{array}$ & $\mathrm{LoS}$ & $\begin{array}{l}\text { The dimensions of the cutting } \\
\text { The height of the cutting } \\
\text { MRS' height relative to the cutting's height }\end{array}$ \\
\hline Hilly terrain & $\begin{array}{l}\text { The surrounding environment is densely scattered with objects } \\
\text { distributed irregularly and non-uniformly. }\end{array}$ & LoS & Density/height of surrounding scatterers \\
\hline Tunnel & $\begin{array}{l}\text { Has unique propagation characteristics different from the } \\
\text { other HST scenarios } \\
\text { Waveguide phenomena is present } \\
\text { Suffers from limited visibility }\end{array}$ & LoS/NLoS & The length, size, and shape of the tunnel \\
\hline Station & Can be classified according to the size or the architecture & LoS/NLoS & $\begin{array}{l}\text { The size of the station } \\
\text { Stations' architecture }\end{array}$ \\
\hline
\end{tabular}

antenna and Rx can suffer $10 \mathrm{~dB}$ greater loss compared with the one between the Tx omni-directional antenna and $\mathrm{Rx}$. The aforementioned results from both measurement campaigns are very useful for the design of HSTs and measurement scenarios. However, more measurements for indoor scenarios in HSTs are needed before confirming that these observations are conclusive. PLs of HST channels in open space and hilly terrain scenarios were reported in [45], [47], [48] and [75], [76], respectively. Measurement data reported in both hilly terrain scenarios showed a breakpoint in the estimated PLs. A dominant and strong LoS component can be easily observed before the breakpoint while the impact of scatterers starts and grows beyond the breakpoint distance. The breakpoint distance depends on the clearance of the first Frensal zone and can be calculated based on the Tx and Rx antenna heights and the wavelength of the transmitted signal [35]. Therefore, different breakpoint distances were reported in the aforementioned hilly terrain measurements, i.e., $778 \mathrm{~m}$ in [75] and $500 \mathrm{~m}$ in [76]. Since viaduct is a common HST scenario, PLs of HST viaduct channels have been thoroughly studied in the literature, e.g., [51], [52], [55]-[58], [62], [64], [65]. Most of these measurements highlighted the impact of the height of the viaduct and the relative height of the BS on the estimated PL. In general, there are two main observations that can be concluded from aforementioned viaduct measurements. First, the higher the BS antenna, the smaller the PL exponent for a given viaduct height. Second, the viaduct reduces the severity of the channel fading. In other words, the higher the viaduct, the less fading severity. Both observations are physically meaningful considering that the increase of the height of the BS and the viaduct over the surrounding obstacles will lead to a clear LoS and reduce the impact of these scatterers on the received signal. The measurements of HST channels in cutting scenarios reported in [69], [70], [72], [73] have demonstrated the impact of the cutting structure on the estimated PLs, i.e., the depth of the cutting and the widths of the top and bottom. A shallow cutting, or low cutting, will lead to a strong LoS condition while deep cutting will lead to a large PL exponent due to the reflections from the cuttings' slopes. A comparison between the PLs of cutting and viaduct scenarios was carried out in [69]. It suggested that the propagation conditions in the cutting scenarios can be worse than those of viaduct ones because of the reflected and scattered components caused by the slopes of the cutting. It is important to note that such conclusion is highly dependant on the dimensions of the studied viaduct and cutting, as we have highlighted the impact of those dimensions on the estimated PLs earlier. On the other hand, shadowing has generally been modeled as lognormal distributed in different HST scenarios. Various channel statistics studied in HST channel measurement campaigns are shown in Table I.

The Ricean $K$-factor is a very important parameter in link budget and channel modeling. Therefore, many papers presented the estimation of $K$-factors in different scenarios, e.g., open space [47], viaduct [51], [53], [54], [62]-[65], cutting [69]-[73], and hilly terrain [75]. The previous discussions of the dominance of the LoS component, the breakpoint distance, and the impact of the viaduct and cutting structure are also related to the $K$-factor. For example, [65] showed how a higher value of the viaduct height will lead to a higher value of the $K$ factor. In the same context, it showed that low viaducts leads to more surrounding scatterers, which leads to an increase in the severity of the fading and results in a considerable fluctuation of the $K$ value. Moreover, the measurement in [65] showed while the $K$-factor is a linear function of distance, the slope $K$ values are different before and after the breakpoint. Similar comprehensive studies on $K$-factors of HST channels but in cutting scenarios were reported in [72], [80]. The analysis showed that wide cuttings increase the possibility of dominant LoS components, which leads to higher $K$-values. Distancedependant linear $K$ models for different cutting dimensions before and after the breakpoint distance were proposed in [72].

In [61], [64], [65], [72], the spatial/temporal variations, e.g., fade depth (FD), level crossing rate (LCR), and average fade duration (AFD), were investigated. FD is a measure of variation in the channel energy about its local mean due to small scale fading and it is calculated from the difference in signal levels between $1 \%$ and $50 \%$. Measurements in viaduct scenarios have showed that FD is independent of the viaduct's height but is affected by the number and closeness 
of surrounding scatterers that are higher than the viaduct [61], [65]. LCR is defined as the expected rate at which the received signal crosses a specified level in a positive-going or negativegoing direction, while AFD is defined as the average period of time for which the received signal is below this specified level, i.e., threshold. LCR and AFD were statistically modeled as functions of the structural parameters of the viaduct and cutting scenarios in [65], [72]. The results showed that the severity of fading in viaduct scenarios is greatly reduced compared with that in open space scenarios, since fewer reflected and scattered paths in viaduct scenarios are expected at the receiver which leads to smaller values of LCR and AFD. Obstacles around the viaduct can cause minor variations of the LCR values but have no significant impact on the AFD. Cutting's dimensions have also very minor impact on the AFD of the received signal while surrounding obstacles and crossing bridges over the cutting have no influence on the LCR and AFD. Doppler behavior and angular information of HST channels in open space scenarios were analyzed in [46] while power delay profiles (PDPs) were investigated in [45], [47], [85], [89], [90]. In [93], a measurement was carried out in a tunnel scenario and the signal propagation characteristics at the breakpoint were discussed.

The stationarity interval, defined as the maximum time duration over which the channel satisfies the wide sense stationary (WSS) condition, of HST channels was investigated in [86] based on measurements. It showed that conventional channel models offered stationary intervals much larger than the actual measured ones. In [92], the non-stationarity of a HST channel in a cutting scenario was investigated using a metric called non-stationarity index. The non-stationarity index was defined as the distance between the auto-correlation of a real time-variant transfer function and the auto-correlation of this transfer function under the WSS assumption. The reported measurement data showed that the non-stationarity index increases when the Doppler frequency shifts varies fast. In the future, more channel statistics, especially those related to small-scale fading parameters, are necessary to be investigated in measurements.

\section{Measurement's Setup Parameters}

Carrier Frequency and Bandwidth: most of the measurement campaigns in the literature were conducted at the carrier frequency of $930 \mathrm{MHz}$ in GSM-R systems [48], [51]-[54], [56]-[59], [61], [64], [65], [69]-[72], [79], [85], [86]. Correspondingly, all of the aforementioned measurements were for narrowband channels with bandwidth of $200 \mathrm{kHz}$. Wideband channel measurements with higher bandwidths, i.e., 10$100 \mathrm{MHz}$, and higher carrier frequencies, i.e., $2.1-5.2 \mathrm{GHz}$, were reported in [42], [44]-[47], [62], [63], [73]-[75], [78], [89]-[91].

Antenna Configuration: The majority of HST measurements campaigns so far have focused on single-input single-output (SISO) systems [42], [44], [45], [48], [51]-[59], [61]-[65], [69]-[75], [78], [79], [85], [86], [89], [90]. Multiple-input multiple-output (MIMO) systems, where multiple antennas are equipped at both ends, are essential for providing higher capacity to meet the requirements of future high speed data transmissions [43]. The channel measurement, particularly the MIMO channel measurement at high moving speeds, remains to be a challenging task. So far, only very few measurement campaigns were conducted using multiple antennas at either the Tx, i.e., single-input multiple-output (SIMO) systems [46], [47], or Rx, i.e., multiple-input single-output (MISO) systems [46]. Hence, HST MIMO wideband channel measurement campaigns with carrier frequency and bandwidth larger than GSM-R ones are needed for future HST communication system developments.

\section{HST CHANNEL MODELS}

HST channel models in the literature can be categorized as large-scale fading models [35], [44], [45], [52], [56]-[58], [62], [65], [69], [70], [73], [75], i.e., PL and shadowing, and small scale-fading models [17], [34], [35], [95], [96], [98], [99], [101]-[105], [109]-[111]. The state-of-the-art of HST channel models has not been investigated yet. Therefore, we will first categorize PL models in Table III. In Table IV, the important HST small-scale fading channel models are briefly reviewed and classified according to the modeling approach, scenario, stationarity, antenna configuration, frequency selectivity (FS), scatterer region, and cellular architecture.

\section{A. Large-Scale Fading Models}

PL estimation is essential for wireless link budget computation and wireless network planning. PL and shadow fading channel models for various HST scenarios have been developed based on measurement results conducted in the open literature [3]-[14]. These PL models are typically expressed as

$$
P L(d)=A+10 n \log _{10}(d),
$$

where $d$ is the distance between the Tx and Rx in unit of meters (m), $n$ is the PL exponent and $A$ is the intercept. Note that SF follows log-normal distributions with the standard deviation for each model is given in Table III.

\section{B. Cellular Architectures and Scenarios}

As mentioned earlier, adopting conventional cellular architecture in HST wireless communication systems may lead to several problems in terms of providing reliable and fast communication to HST passengers. Therefore, other cellular architectures, such as DAS, CoMP, and MRS need to be considered. In the literature, most of the proposed channel models have considered the conventional architecture where fixed BSs are installed on the track-side to provide wireless coverage to HST passengers inside carriages [17], [95], [96], [98], [99], [101], [102], [109]. By considering MRS solution, we will have two channels, outdoor channel between the BS and the MRS and an indoor one between MRS and train passengers. The properties of radio channels in the carriages resemble indoor environment and hence they can be modeled using existing indoor channel models [44]. Therefore, [34], [35], [103]-[105], [111] have focused on modeling the outdoor 
TABLE III

PL AND SHADOW FADING MODELS FOR HST CHANNELS.

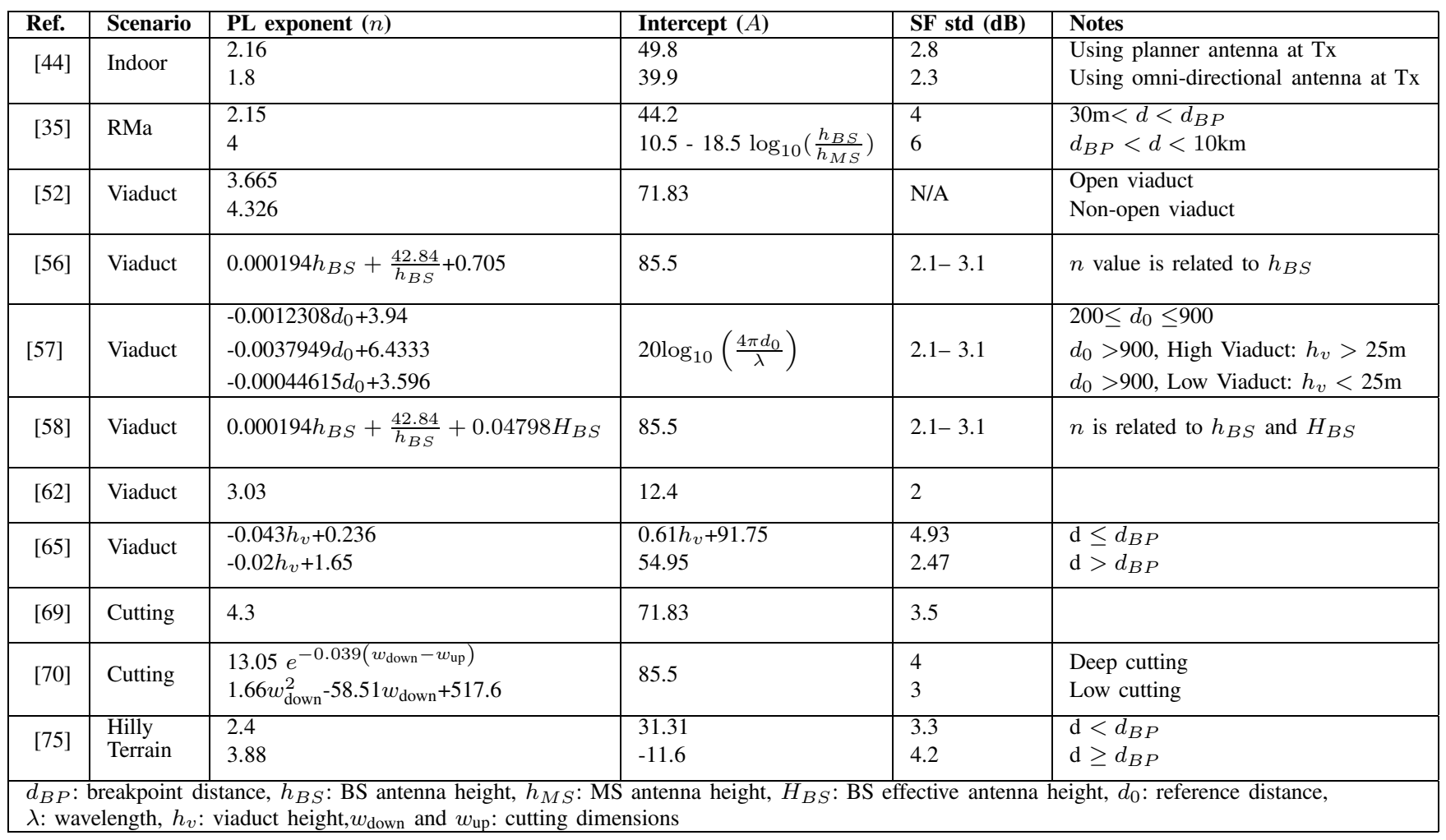

TABLE IV

IMPORTANT HST SMALL-SCALE FADING CHANNEL MODELS.

\begin{tabular}{|c|c|c|c|c|c|c|c|c|}
\hline Ref. & Channel & Channel Model & Scenario & Stationarity & Antenna & FS & Scatterer Region & Cellular Architecture \\
\hline$[17]$ & Outdoor & Non-fading & Open space & Stationary & MIMO & Narrowband & N/A & Conventional \\
\hline [95] & Outdoor & GBDM & Open space & Non-stationary & MIMO & Wideband & Non-isotropic & Conventional \\
\hline [96] & Outdoor & GBDM & Open space & Non-stationary & MISO & Wideband & Non-isotropic & Conventional \\
\hline [97] & Outdoor & GBDM & Various & Non-stationary & SISO & Narrowband & Non-isotropic & Conventional \\
\hline [98], [99] & Outdoor & GBDM & Tunnel & Non-stationary & SISO & Narrowband & Non-isotropic & Conventional \\
\hline$[101]$ & Outdoor & RS-GBSM & Open space & Stationary & MIMO & Narrowband & Non-isotropic & Conventional \\
\hline [102] & Outdoor & RS-GBSM & Open space & Stationary & MIMO & Wideband & Isotropic & Conventional \\
\hline [103]-[105] & Outdoor & RS-GBSM & Open space & Non-stationary & MIMO & Wideband & Non-isotropic & Mobile Relay \\
\hline [106] & Outdoor & GBSM & various & Non-stationary & MIMO & Wideband & Non-isotropic & Mobile Relay \\
\hline$[34],[35]$ & Outdoor & IS-GBSM & Open space & Stationary & MIMO & Wideband & Non-isotropic & Mobile Relay \\
\hline [101], [108] & Outdoor & IS-GBSM & Cutting & Stationary & MIMO & Narrowband & Isotropic & Conventional \\
\hline [91] & Outdoor & IS-GBSM & Open space & Non-stationary & MIMO & Wideband & Non-isotropic & Conventional \\
\hline [109], [110] & Outdoor & NGSM & Open space & Non-stationary & MIMO & Wideband & Non-isotropic & Conventional \\
\hline$[111]$ & Outdoor & NGSM & Viaduct & Non-stationary & MIMO & Wideband & Non-isotropic & Mobile Relay \\
\hline
\end{tabular}

channel because of the challenges that this channel faces due to the high velocity of the Rx.

HST scenarios have been presented in details earlier in this paper in Section II. While most of these scenarios can only be encountered in railway environments, open space scenario is similar to the rural or urban scenarios that can be found in conventional V2I or V2V communication systems. Therefore, most of the current HST channel models, developed from V2I and $\mathrm{V} 2 \mathrm{~V}$ channel models by taking into account the effect of the high velocity of the Rx on the channel parameters, have been proposed for open space scenario [17], [34], [35], [95], [96], [102]-[105], [109]. Channel models for tunnel, cutting, and viaduct scenarios were studied in [99], [101], and [111], respectively.
In summary, more HST channel models that consider other cellular architectures, such as DAS, are needed in the future. In addition, more HST scenarios should be considered in proposing future HST channel models.

\section{Modeling Approaches of HST Small-Scale Fading Models}

In terms of modeling approach, the current HST channel models in the literature, presented in Table IV, can be classified as deterministic [95]-[99] and stochastic channel models. The latter can be further classified into geometrybased stochastic models (GBSMs) [34], [35], [101]-[105] and non-geometrical stochastic models (NGSMs) [109], [111] as illustrated in Fig. 3. 


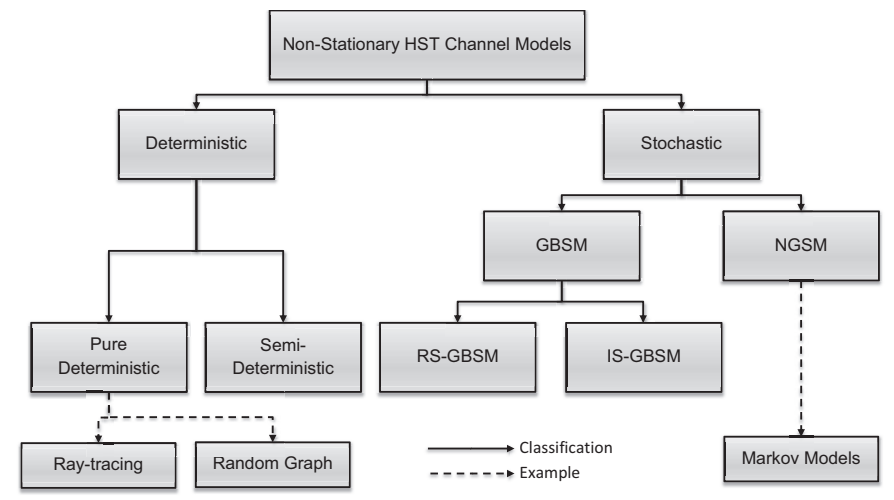

Fig. 3. Classification of non-stationary HST channel models

1) Deterministic channel models: Deterministic channel models are usually based on the detailed description of specific propagation environment and antenna configuration. The amplitudes, phases and delays of the propagated waves are obtained using intensive simulations that incorporate details of propagation environments like, roads, building, trees, houses, ...etc. Therefore, deterministic models are physically meaningful and potentially accurate. Geometry-based deterministic models (GBDMs) based on ray-tracing method were proposed in [96]-[99] to model HST propagation channels in different HST scenarios. In [99], a three-dimensional (3D) ray-tracing approach for wave propagation modeling in HST tunnels was presented. The proposed model resulted in a complex channel impulse response that incorporates channel information, e.g., the wave-guide effect observed in tunnels and the impact of another train passing in the opposite direction on the Doppler shift and time delay. Authors of [97] implemented similar approach to model HST channels in various scenarios. Both of [99] and [97] have used measurement results to verify the proposed channel models. Another HST channel model based on 3D ray-tracing approach has been implemented in [96] to analyze the channel characteristic, e.g., the frequencyselectivity and time-variance (Doppler spread). The objects, e.g., trees, buildings, or barriers, on both sides of the railway track were modeled using rectangular boxes with scattering wave propagation characteristics where the dimensions of the boxes are statistically generated. Since the propagation characteristics of electromagnetic (EM) waves in tunnels are significantly different from those in other HST environments, a multi-mode waveguide channel model is proposed in [113]. The proposed model, which is a hybrid model that combines the geometrical optical model and waveguide model, can characterize the wave propagation both in near and far regions of the source. However, the aforementioned model failed to discuss the far LoS (FLOS) phenomena observed inside tunnels [114] or provide a mechanism to determine the breakpoint for different propagation regions in tunnels [6]. A GBDM based on random propagation-graph was proposed in [95] to characterize time-variant HST channels in open space scenario. Similar to ray-tracing method, propagationgraph can predict channel impulse responses by thorough searching for propagation paths that connect $\mathrm{Tx}$ and $\mathrm{Rx}$. This modeling approach can be performed by considering the geometry of the simulated environments, e.g., the distribution, mobility, and visibility of the scatterers. Despite their high accuracy, GBDMs require detailed descriptions of the propagation environments and extensive computational resources to be implemented. To avoid the high complexity of implementing GBDMs while maintaining sufficient accuracy, semi-deterministic models for viaduct and cutting scenarios of HST were proposed in [100]. However, the proposed models have only considered large-scale fading, i.e., PL and SF, and neglected the effect of small-scale fading parameters on the received signal.

2) GBSMs: In GBSMs, the impulse responses of HST channels are characterized by the law of wave propagation applied to specific Tx, Rx, and scatterer geometries which are predefined in a stochastic fashion according to certain probability distributions. Different types of GBSMs differ mainly in the proposed scatterer distributions. Based on the position of the effective scatterers, GBSMs can be further classified as regular-shaped GBSMs (RS-GBSM) such as onering [102], two-ring, and ellipses [103]-[105], and irregular shaped GBSMs (IS-GBSMs) [34], [35], [101].

RS-GBSMs assume that all the effective scatterers are placed on regular shapes, and therefore different RS-GBSMs have different shapes of scatterer distributions, e.g., onering, two-ring, and ellipses for two-dimensional (2D) models and one sphere, two-spheres, elliptic-cylinders for 3D ones. RS-GBSMs often result in closed-form solutions or at least mathematically tractable formulas. The generalized principle of designing RS-GBSM follows the following steps. First, a geometrical model is adopted assuming that scatterers are located on regular shapes (e.g., one-ring, two-ring, and ellipse). Then, a stochastic reference model with an infinite number of scatterers is developed based on the adopted geometrical model. However, the reference model cannot be used for simulations and therefore a corresponding simulation model with a finite number of effective scatterers is needed. The parameters of the simulation model is computed by using a proper parameter computation method, e.g., the extended method of exact Doppler spread (EMEDS), modified method of equal area (MMEA), or the $L_{p}$-Norm Method (LPNM) [107]. In [102], a one-ring RS-GBSM was proposed to model HST channel in open space scenario. The scatterers are assumed to be distributed on a ring around the MS where different PDF of the scatterers were analyzed. Considering the narrowband GSM-R for HST communication system, a 3D one-sphere RS-GBSM was proposed in [101] for open space scenario. The proposed model used Von Mises distribution to describe the azimuth angles and the space-time (ST) crosscorrelation function (CCF) was derived. However, both of the aforementioned models have assumed that the HST channel satisfies the WSS condition that has been proved incorrect by measurements [46]. To fill in this gap, non-stationary RSGBSMs were proposed in [103]-[106] for wideband MIMO HST channels considering the deployment of MRS on the top of the train. Fig. 4 illustrates the proposed RS-GBSMs, which consist of multiple confocal ellipses with single-bounced rays 


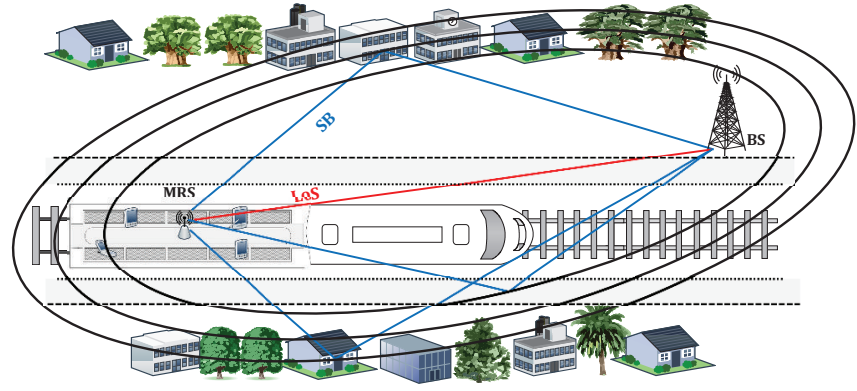

Fig. 4. A HST communication system in a cutting scenario with LoS and single-bounced rays.

and the LoS component.

The model was first introduced in [103], [104] where it considered the distance between the $\mathrm{Tx}$ and $\mathrm{Rx}$ as timevarying to capture the non-stationarity of the HST channel. Then, the model has been further developed in [105] by considering other time-varying model parameters, i.e., angles of departure (AoDs) and angles of arrival (AoAs). By adopting some key scenario-specific channel parameters, this model was further extended in [106] to be applicable to the three most common HST scenarios, i.e., open-space, viaduct, and cutting scenarios [36], and hence is the first generic HST channel model. To demonstrate its applicability, the proposed generic non-stationary HST channel model was verified by measurements in terms of stationary time for the open space scenario and the ACF, LCR, and stationary distance for the viaduct and cutting scenarios [106].

IS-GBSMs place the effective scatterers with predefined properties at random locations with certain statistical distributions usually obtained/approximated from measurements [115]. Unlike RS-GBSMs, the random locations of the scatterers do not form regular shapes and the signal contributions of the effective scatterers are determined from a greatlysimplified ray-tracing method and finally the total signal is summed up to obtain the complex impulse response. ISGBSMs for HST channels have been introduced in the RMa scenario in WINNER II [35] and moving networks scenario in IMT-A channel models [34] with the train speed can be up to $350 \mathrm{~km} / \mathrm{h}$ and the MRS technology is employed. In [101], IS-GBSM has been proposed for HST channel in cutting scenario assuming the scatterers to be uniformly distributed on the surface of the two slopes of the cutting. However, the aforementioned channel models have neglected the nonstationarity of HST channels and assumed that the WSS assumption can still be applied. Moreover, GBSMs are very complex for upper-layer protocol design and performance analysis and less complex channel models are preferred.

3) NGSMs: NGSMs characterize physical parameters of a HST propagation channel in a completely stochastic manner by providing their underlying probability distribution functions without assuming an underlying geometry. An NGSM based on finite-state Markov chains for HST wireless communication channels was proposed in [109]. The proposed model is able to capture the characteristics of time-varying HST wireless channel by using Markov chain to track the channel state varia- tion at different received signal-to-noise ratio (SNR) intervals. However, the model has not been verified by using real-field measurements and thus deserves more investigation. Authors of [111] have followed similar approach to model the dynamic evolution of multi-path components, i.e., birth-death process, using four-state Markov chain model. The four proposed states are no birth/death, births only, deaths only, and both births and deaths. The transition matrix of the birth-death process was calculated based on the measurement presented in [62]. Based on measurement of HST channels in viaduct and cutting scenarios, a finite-state Markov channel was also proposed in [112]. Simulation results showed that Ricean distribution can well characterize the measured amplitude of the small-scale fading in both HST scenarios and that NGSM can effectively capture the dynamic nature of the fast fading in HST channels.

\section{RESEARCH DiRECTIONS IN HST CHANNEL MEASUREMENTS AND MODELS}

In this section, we will discuss important research directions that can be considered as guidelines for conducting future HST measurement campaigns and developing more realistic HST channel models.

\section{A. Non-Stationarity of HST Channels}

Measurements in the literature have demonstrated that HST channels are non-stationary since the stationary conditions, measured by stationary interval, retain to a very short period of time in comparison with other types of channels, e.g., V2I and V2V channels [86]. This is mainly caused by the very high speed of the trains and the encountered changes in surrounding areas. Although the non-stationarity of HST channels has been implicitly considered in GBDMs [95]-[97], [99], but these models are mainly site-specific and cannot be easily generalized to a variety of scenarios. The nonstationarity feature of HST channels has been considered in NGSM proposed in [109] by implementing the birth-death process to simulate the appearance and disappearance of the scatterers, and in RS-GBSMs in [103]-[106] by providing the time-variant functions of several channel model parameters, i.e., angular parameters, Doppler frequency, Ricean $K$-factor, and the distance between $\mathrm{Tx}$ and Rx. However, verifying the proposed models by real-field measurements was only performed in [106] and therefore more comprehensive investigations in future are required to validate the accuracy of those models. Future non-stationary channel models should consider more time-variant model parameters, such as cluster powers and delays and also investigate the effect of the drift of scatterers into different delay taps on the non-stationarity of HST channels and the resulting correlation between these taps.

\section{B. Statistical Properties of HST channels}

Investigating the statistical properties of HST channels is essential for understanding and analyzing the HST communication system. In Table I, several channel statistics obtained from measurements were presented. However, most of proposed HST channel models in the literature have failed to 
provide the corresponding theoretical analysis. In [101], the ST CCF was derived based on the proposed stationary narrowband HST channel model. In [105], a novel theoretical framework that characterizes non-stationary mobile fading channels in terms of their system functions and correlation functions was proposed. Based on this theoretical framework, different time-variant statistical properties of the RS-GBSMs in [105], [106] were derived, i.e., time-variant space CCFs, timevariant autocorrelation functions (ACFs), time-variant spaceDoppler (SD) PSDs, local scattering functions (LSFs) [105], and LCRs [106]. It is highly desirable to investigate the statistical properties of other HST channel models and further develop the aforementioned theoretical framework to include more statistical properties.

\section{HST Scenarios}

HST scenarios were classified and thoroughly explained in Section II of this article. During its travel and due to its high velocity, HST runs across diverse scenarios so rapidly that a single model is incapable of capturing accurately the variations of HST channels. While most standard channel models did not consider any of the HST scenarios, IMTA channel model, WINNER II channel model, and most of the non-standard HST channel models were proposed for open space scenario only. Due to the unique environment of tunnels, the propagation characteristics of signals inside tunnels are different from those of other HST scenarios. Conventional channel modeling techniques suitable for other HST scenarios are not directly applicable to tunnel scenarios. Moreover, research on channel modeling inside tunnels is yet to solve main problems such as the accurate characterization of multimode waveguide propagation and the determination of breakpoint for different propagation mechanisms inside tunnels. In addition, the research on 3D channel models for tunnels is still in its very early stages and accurate generic channel models that can be applied to different types of tunnel channels are still missing in the literature. Therefore, channel characterization and modeling for tunnel scenario are still a quite challenging topic and needs to be further investigated. Moreover, it is essential that future HST channel models consider other scenarios such as station scenarios that also have their unique channel characteristics, and preferably take into account the impact of diverse scenarios on HST channels.

\section{3D HST Channel Models}

Apart from the GBDMs that use 3D ray-tracing tool to model HST channels [96]-[99], HST channels were generally proposed and measured assuming that propagation waves are traveling in two dimensions and therefore ignore the impact of the elevation angle on channel statistics. In reality, radio waves propagate in three dimensions and scatterers are disperse in elevation, i.e., vertical plane, as well as in azimuth, i.e., the horizontal plane. Recently, the 3GPP has developed a 3D channel model in urban microcell and urban macrocell scenarios following the framework of WINNER II channel model [116]. The proposed 3D 3GPP channel model introduced the zenith AoD and zenith AoA that are modeled by inverse Laplacian functions [117]. The 3D extensions of SCM and the WINNERII/WINNER+ channel models were proposed in [118] and [119], respectively, and an extension of the IMTA channel model to the elevation plane was proposed in [120], [121]. However, none of the aforementioned channel models has considered any of the HST scenarios. Thus, 3D channel measurements and models are necessary, especially when the HST is close to the BS where considering elevation angles can demonstrate the impact of the waves reflected from ground on the received signal.

\section{E. HST-to-HST Communication}

HST-to-HST communication is proposed to enable HSTs exchange controlling and traffic information such as road obstacles, accidents via the wireless communication links. A HST-to-HST communication model based on multihop and cooperation is proposed in [122]. In the proposed model, a source train uses trains on other tracks as relays to transmit signals to destination train on the same track. Based on proposed HST-to-HST channel model, the bit error rate (BER) performance was investigated in [122] using suburban scenario of COST 207 channel model and the outage capacity was analyzed in [123] using Nakagami- $m$ channel model. Despite its importance as a safety measure to avoid accidents, the development of HST-to-HST channels is still in its preliminary phase and further investigations are required.

\section{F. System Performance}

Investigating the performance of HST communication systems is the basis for system design and network planning. In [26], the HST communication system performance was investigated using data throughput to evaluate a seamless dual-link handover scheme. Another handover scheme was proposed in [27] and the system performance was evaluated by tracking the changes of throughput and signal to noise and interference ratio (SINR) over the time. The changes of SINR over the HST velocity has been investigated in [124] to evaluate a transmit beamforming algorithm proposed for canceling the inter-channel-interference (ICI) in HST communication systems. The performance of HST communication system that implements beamforming technique was also evaluated in [125] using measured throughput, SINR, and received signal strength indicator level. The implementation of DAS in HST communication system was evaluated in [19] by using spectrum efficiency as a system performance metric. In [126], BER was used to evaluate a proposed radio resource allocation scheme for OFDMA HST systems. BER performance of a HST communication system was also investigated in [127] where beamforming and Alamouti combined downlink transmission schemes were proposed. Mobile broadband performance experiences from regional HSTs were investigated in [128] by monitoring the fluctuation of system throughput caused by the varying distance between the BS and HST, multi-path fading and co-channel interference conditions. A temporal proportional fair power allocation scheme for HST wireless communication systems has been proposed in 
[129]. The proposed scheme is designed to achieve a tradeoff between power efficiency and fairness along the time. HST channel capacity was analyzed in [130] to study the impact of different antenna array configurations on MIMO HST communication systems. More comprehensive system performance analysis that evaluates other schemes and considers more system performance indicators, e.g., capacity and quality of service (QoS), is required in the future.

\section{CONCluding Remarks}

This article has provided a survey of HST channels, that represent high-mobility V2I channels, in terms of conducted measurements and proposed channel models. We have classified HST channel measurements according to scenarios, carrier frequencies, bandwidths, measured channels, antenna configurations, train speeds, and channel statistics. We have also presented various HST large-scale fading channel models in the literature. Then, we have classified HST small-scale fading channel models based on their modeling approaches, scenarios, stationarity, FS, and cellular architecture. Finally, we have highlighted some research directions in HST channel measurements and modeling. The discussions here will hopefully shed lights on recent challenges that face HST channels and help in conducting future measurement campaigns and developing more practical HST channel models.

\section{REFERENCES}

[1] C.-X. Wang, F. Haider, X. Gao, X.-H. You, Y. Yang, D. Yuan, H. Aggoune, H. Haas, S. Fletcher, E. Hepsaydir, "Cellular architecture and key technologies for 5G wireless communication networks," IEEE Commun. Mag., vol. 52, no. 2, pp. 122-130, Feb. 2014.

[2] A. F. Molisch, F. Tufvesson, J. Karedal, and C. F. Mecklenbrauker, "A survey on vehicle-to-vehicle propagation channels," IEEE Trans. Wireless Commun., vol. 16, no. 6, pp. 12-22, Dec. 2009.

[3] C.-X. Wang, X. Cheng, and D. I. Laurenson, "Vehicle-to-vehicle channel modeling and measurements: recent advances and future challenges", IEEE Commun. Mag., vol. 47, no. 11, pp. 96-103, Nov. 2009.

[4] F. Abrishamkar and J. Irvine, "Comparison of current solutions for the provision of voice services to passengers on high speed trains," in Proc. IEEE VTC'O0-Fall, Boston, USA, Sept. 2000, pp. 1498-1505.

[5] D. T. Fokum and V. S. Frost, "A survey on methods for broadband internet access on trains," IEEE Commun. Surveys Tuts, vol. 12, no. 2, pp. 171185, 2nd Quarter 2010.

[6] B. Ai, X. Cheng, T. Kurner, Z.-D. Zhong, K. Guan, R.-S. He, L. Xiong, D. W. Matolak, D. G. Michelson, and C. Briso-Rodriguez, "Challenges toward wireless communications for high-speed railway," IEEE Trans. Intell. Transp. Syst., vol. 15, no. 5, pp. 2143-2158, Oct. 2014.

[7] M. Goller, "Application of GSM in high speed trains: measurements and simulations," IEE Colloquium on Radiocommunications in Transp., pp. 1-7, May 1995.

[8] C. Briso, C. Cortes, F. J. Arques, and J. I. Alonso, "Requirements of GSM technology for the control of high speed trains," in Proc. IEEE PIMRC'02, Lisbon, Portugal, Sept. 2002, pp. 792-793.

[9] K. Kastell, S. Bug, A. Nazarov, and R. Jakoby, "Improvments in railway communication via GSM-R," in Proc. IEEE VTC'06-Spring, Melbourne, Australia, May 2006, pp. 3026-3030.

[10] J. Calle-Sńnchez, M. Molina-Garcia, J. I. Alonso, and A. FerníndezDurn, "Long term evolution in high speed railway environments: Feasibility and challenges," Bell Labs Techn. J., vol. 18, no. 2, pp. 237-253, Sept. 2013.

[11] K. Guan, Z. Zhong, and B. Ai, "Assessment of LTE-R using high speed railway channel model," in Proc. CMC'11, Qingdao, China, Apr. 2011, pp. 461-464.

[12] J. Rodriguez-Pineiro, P. Suarez-Casal, M. Lerch, S. Caban, J. A. GarciaNaya, L. Castedo, and M. Rupp, "LTE downlink performance in high speed trains," in Proc. IEEE VTC'15-Spring, Glasgow, Scotland, May 2015, pp. 1-5.
[13] S. Zhang, M. Wu, and X. Lin, "Results and analysis based on a novel LTE-R channel measurement at $2.6 \mathrm{GHz}$," in Proc. ICT'15, Sydney, Australia, Apr. 2015, pp. 403-407.

[14] M. Cheng, X. Fang, and W. Luo, "Beamforming and positioning-assisted handover scheme for long-term evolution system in high-speed railway," IET Communications, vol. 6, no. 15, pp. 2335-2340, Oct. 2012.

[15] M. Alasali and C. Beckman, "LTE MIMO performance measurements on trains," in Proc. EuCAP'13, Gothenburgh, Sweden, Apr. 2013, pp. 2327-2330.

[16] F. J. Martin-Vega, I. M. Delgado-Luque, F. Blanquez-Casado, G. Gomez, M. C. Aguayo-Torres, and J. T. Entrambasaguas, "LTE performance over high speed railway channel," in Proc. IEEE VTC'13-Fall, Las Vegas, USA, Sept. 2013, pp. 1-5.

[17] 3GPP, TS36.104, V11.3.1, "3rd Generation Partnership Project; Technical Specification Group Radio Access Network; Evolved Universal Terrestrial Radio Access (E-UTRA); Base Station (BS) radio transmission and reception (Release 11)," Jan. 2013.

[18] K. Yamada, Y. Sakai, T. Suzuki, Y. Kawahara, T. Asami, and H. Aida, "A communication system with a fast handover under a high speed mobile environment," in Proc. IEEE VTC'10-Fall, Ottawa, Canada, Sept. 2010, pp. $1-5$.

[19] J. Wang, H. Zhu, and N. J. Gomes, "Distributed antenna systems for mobile communications in high speed trains," IEEE J. Sel. Areas Commun., vol. 30, no. 4, pp. 675-683, May 2012.

[20] H.-A. Hou and H.-H. Wang, "Analysis of distributed antenna system over high-speed railway communication," in Proc. IEEE PIMRC'12, Sydney, Australia, Sept. 2012, pp. 1300-1305.

[21] C. Yang, L. Lu, C. Di, and X. Fang, "An on-vehicle dual-antenna handover scheme for high-speed railway distributed antenna system," in Proc. IEEE WiCOM'10, Chengdu, China, Sept. 2010, pp. 1-4.

[22] D. Lee, H. Seo, B. Clerckx, E. Hardouin, D. Mazzarese, S. Nagata, and K. Sayana, "Coordinated multipoint transmission and reception in LTE-advanced: deployment scenarios and operational challenges," IEEE Commun. Mag., vol. 50, no. 2, pp. 148-155, Feb. 2012.

[23] C.-X. Wang, X. Hong, X. Ge, X. Cheng, G. Zhang, and J. S. Thompson, "Cooperative MIMO channel models: a survey," IEEE Commun. Mag., vol. 48, no. 2, pp. 80-87, Feb. 2010.

[24] Y. Zhou, Z. Pan, J. Hu, J. Shi, and X. Mo, "Broadband wireless communications on high speed trains," in Proc. IEEE WOCC'11, New Jersey, USA, Apr. 2011, pp. 1-6.

[25] L. Tian, Y. Zhou, J. Li, Y. Huang, J. Shi, and J. Zhou, "A novel handover scheme for seamless wireless connectivity in high-speed rail," in Proc. IEEE WiMob'11, Wuhan, China, Oct. 2011, pp. 230-236.

[26] L. Tian, J. Li, Y. Huang, J. Shi, and J. Zhou, "Seamless dual-link handover scheme in broadband wireless communication systems for highspeed rail," IEEE J. Sel. Areas Commun., vol. 30, no. 4, pp. 708-718, May 2012.

[27] O. B. Karimi, L. J. Liu, and C. Wang, "Seamless wireless connectivity for multimedia services in high speed trains," IEEE J. Sel. Areas Commun., vol. 30, no. 4, pp. 729-739, May 2012.

[28] F. Haider, C.-X. Wang, H. Haas, D. Yuan, H. Wang, X. Gao, X.-H. You, and E. Hepsaydir, "Spectral efficiency analysis of mobile femtocell based cellular systems," in Proc. IEEE ICCT'11, Jinan, China, Sept. 2011, pp. 347-351.

[29] F. Haider, C.-X. Wang, B. Ai, H. Haas, and E. Hepsaydir, "Spectralenergy efficiency trade-off of cellular systems with mobile femtocell deployment," IEEE Trans. Veh. Technol., accepted for publication.

[30] X. Qian, H. Wu, and J. Meng, "A dual-antenna and mobile relay station based handover in distributed antenna system for high-speed railway," in Proc. IEEE IMIS'13, Taichung, Taiwan, July 2013, pp. 585-590.

[31] W. Luo, R. Zhang, and X. Fang, "A CoMP soft handover scheme for LTE systems in high speed railway," EURASIP J. Wireless Commun. Networking, vol. 2012, 9 pages, 2012. doi:10.1186/1687-1499-2012-196.

[32] B. Lannoo, D. Colle, M. Pickavet, and P. Demeester, "Radio-over-fiberbased solution to provide broadband internet access to train passengers," IEEE Commun. Mag., vol. 45, no. 2, pp. 56-62, Feb. 2007.

[33] J.-Y. Zhang, Z.-H. Tan, and X.-X. Yu, "Coverage efficiency of radioover-fiber network for high-speed railways," in Proc. IEEE WiCOM'10, Chengdu, China, Sept. 2010, pp. 1-4.

[34] ITU-R M.2135-1, "Guidelines for Evaluation of Radio Interface Technologies for IMT-Advanced," Geneva, Switzerland, Rep. ITU-R M.21351, Dec. 2009.

[35] P. Kyösti, et al., "WINNER II channel models," IST-4-027756, WINNER II D1.1.2, v1.2, Apr. 2008.

[36] R. He, Z. Zhong, B. Ai, and K. Guan, "Reducing the cost of high-speed railway communications: From the propagation channel view," IEEE Trans. Intell. Transp. Syst., vol. 16, no. 4, pp. 2050-2060, Aug. 2015. 
[37] B. Ai, R. He, Z. Zhong, K. Guan, B. Chen, P. Liu, and Y. Li. "Radio wave propagation scene partitioning for high-speed rails," Int. J. Antennas Propag., vol. 2012, Article ID 815232, 7 pages, 2012. doi: $10.1155 / 2012 / 815232$

[38] "Universal Mobile Telecommunications System (UMTS); Radio Frequency (RF) system scenarios (Release 12)," 3GPP TR 25.942, Oct. 2014, version 12.0.0.

[39] L. Liu, J. Poutanen, F. Quitin, K. Haneda, F. Tufvesson, P. De Doncker, P. Vainikainen, and C. Oestges, "The COST 2100 MIMO channel model," IEEE Wireless Commun., vol. 19, no. 6, pp. 92-99, Dec. 2012.

[40] "Requirements for the Radio Interface(s)for International Mobile Telecommunications-2000 (IMT-2000)," ITU, Geneva, Switzerland, Tech. Rep. ITU-R M.1034-1, 2000.

[41] J. Rodríguez-Piñeiro, J. A. García-Naya, Á. Carro-Lagoa, and L. Castedo, "A testbed for evaluating LTE in high-speed trains," in Proc. IEEE DSD'13, Santander, Spain, Sept. 2013, pp. 175-182.

[42] N. Kita, T. Ito, S. Yokoyama, M-C. Tseng, Y. Sagawa, M. Ogasawara, and M. Nakatsugawa, "Experimental study of propagation characteristics for wireless communications in high-speed train cars," in Proc. EuCAP'09, Berlin, Germany, Mar. 2009, pp. 897-901.

[43] J. Yang, B. Ai, and Z. Zhong "Construction and capacity analysis of high-rank LoS MIMO channels in high speed railway scenarios," Int. J. Antennas Propag., vol. 2012, Article ID 423759, 7 pages, 2012. doi:10.1155/2012/423759.

[44] W. Dong, G. Liu, L. Yu, H. Ding, and J. Zhang, "Channel properties of indoor part for high-speed train based on wideband channel measurement," in Proc. CHINACOM'10, Beijing, China, Aug. 2010, pp. 1-4.

[45] M. Zhao, M. Wu, Y. Sun, D. Yu, S. Di, P. Zhou, X. Zeng, and S. $\mathrm{Ge}$, "Analysis and modeling for train-ground wireless wideband channel of LTE on high-speed railway," in Proc. IEEE VTC'13-Spring, Dresden, Germany, June 2013, pp. 1-5.

[46] R. Parviainen, P. Kyosti, Y. Hsieh, P. Ting, and J. Chiou, "Results of high speed train channel measurements," in COST 2100 TD'08, Lille, France, Oct. 2008.

[47] K. Pekka, "WINNER II channel models part II radio channel measurement and analysis results," IST-4-027756, WINNER II D1.1.2, v1.0, Sept. 2007

[48] R. He, A. F. Molisch, Z. Zhong, B. Ai, J. Ding, R. Chen, and Z. $\mathrm{Li}$, "Measurement based channel modeling with directional antennas for high-speed railways," in Proc. IEEE WCNC'13, Shanghai, China, Apr. 2013, pp. 2932-2936.

[49] M. Dohler and Y. Li, Cooperative Communications: Hardware, Channel \& PHY. Chippenham: Wiley, 2010.

[50] D. Wang, L. Song, X. Kong, and Z. Zhang, "Near-ground path loss measurements and modeling for wireless sensor networks at $2.4 \mathrm{GHz}$," Int. J. Distributed Sensor Net., vol. 2012, Article ID 969712, 10 pages, 2012. doi:10.1155/2012/969712.

[51] H. Wei, Z. Zhong, K. Guan, and B. Ai, "Path loss models in viaduct and plain scenarios of the high-speed railway," in Proc. CHINACOM'10, Beijing, China, Aug. 2010, pp. 1-5.

[52] J. Lu, G. Zhu, and B. Ai, "Radio propagation measurements and modeling in railway viaduct area," in Proc. IEEE WiCOM'10, Chengdu, China, Sept. 2010, pp. 1-5.

[53] L. Gao, Z. Zhong, B. Ai, and L. Xiong, "Estimation of the Ricean factor in K the high speed railway scenarios," in Proc. CHINACOM'10, Beijing, China, Aug. 2010, pp. 1-5.

[54] R. He, Z. Zhong, B. Ai, and J. Ding, "Distance-dependent model of Ricean K-factors in high-speed rail viaduct channel," in Proc. IEEE VTC'12-Fall, Québec City, Canada, Sept. 2012, pp. 1-5.

[55] R. He, Z. Zhong, and B. Ai, "Path loss measurements and analysis for high-speed railway viaduct scene," in Proc. IWCMC'10, Caen, France, July 2010, pp. 266-270.

[56] R. He, Z. Zhong, B. Ai, L. Xiong, and H. Wei, "A novel path loss model for high-speed railway viaduct scenarios," in Proc. IEEE WiCOM'11, Wuhan, China, Sept. 2011, pp. 1-4.

[57] R He, Z. Zhong, B. Ai, L. Xiong, and J. Ding, "The effect of reference distance on path loss prediction based on the measurements in high-speed railway viaduct scenarios," in Proc. CHINACOM'11, Harbin, China, Aug. 2011, pp. 1201-1205.

[58] R. He, Z. Zhong, B. Ai, and J. Ding, "An empirical path loss model and fading analysis for high-speed railway viaduct scenarios," IEEE Antennas Wireless Propag. Lett., vol. 10, pp. 808-812, Aug. 2011.

[59] H. Wei, Z. Zhong, L. Xiong, B. Ai, and R. He, "Study on the shadow fading characteristic in viaduct scenario of the high-speed railway," in Proc. CHINACOM'11, Harbin, China, Aug. 2011, pp. 1216-1220.
[60] B. Zhang, Z. Zhong, B. Ai, D. Yao, and R. He, "Measurements and modeling of cross-correlation property of shadow fading in high-speed railways," in Proc. IEEE VTC'14-Fall, Vancouver, Canada, Sept. 2014, pp. $1-5$.

[61] L. Gao, Z. Zhong, B. Ai, L. Xiong, and H. Zhang, "Analysis and emulation of the small-scale fading characteristics in the high-speed rail scenarios," in Proc. CHINACOM'11, Harbin, China, Aug. 2011, pp. 1181-1185.

[62] L. Liu, C, Tao, J, Qiu, H. Chen, L. Yu, W. Dong, and Y. Yuan, "Positionbased modeling for wireless channel on high-speed railway under a viaduct at $2.35 \mathrm{GHz}$," IEEE J. Sel. Areas Commun., vol. 30, no. 4, pp. 834-845, May 2012.

[63] Y. Guo, J. Zhang, C. Zhang, and L. Tian, "Correlation analysis of highspeed railway channel parameters based on channel measurement," in Proc. HMWC'13, Shanghai, China, Nov. 2013, pp. 1-5.

[64] R. He, Z. Zhong, B. Ai, and J. Ding, "Measurements and analysis of short-term fading behavior for high-speed rail viaduct scenario," in Proc. IEEE ICC'12, Ottawa, Canada, June 2012, pp. 4563-4567.

[65] R. He, Z. Zhong, B. Ai, G. Wang, J. Ding, and A. F. Molisch, "Measurements and analysis of propagation channels in high-speed railway viaducts," IEEE Trans. Wireless Commun., vol. 12, no. 2, pp. 794-805, Feb. 2013.

[66] Q. Wang, C. Xu, M. Zhao, and D. Yu "Results and analysis for a novel $2 \times 2$ channel measurement applied in LTE-R at $2.6 \mathrm{GHz}$," in Proc. IEEE WCNC'14, Istanbul, Turkey, Apr. 2014, pp. 177-181.

[67] R. Sun, C. Tao, L. Liu, Z. Tan, L. Zhang, and T. Zhou, "Nonisotropic scattering characteristic in an alternant tree-blocked viaduct scenario on high-speed railway at $2.35 \mathrm{GHz}$, , Int. J. Antennas and Propag., vol. 2014, Article ID 642894, 9 pages, 2014. doi:10.1155/2014/642894.

[68] T. Zhou, C. Tao, L. Liu, and Z. Tan, "A semiempirical MIMO channel model in obstructed viaduct scenarios on high-speed railway," Int. J. Antennas and Propag., vol. 2014, Article ID 287159, 10 pages, 2014. doi:10.1155/2014/287159.

[69] J. Lu, G. Zhu, and C. Briso-Rodriguez, "Fading characteristics in the railway terrain cuttings," in Proc. IEEE VTC'11-Spring, Budapest, Hungary, May 2011, pp. 1-5.

[70] R. He, Z. Zhong, B. Ai, and J. Ding, "Propagation measurements and analysis for high-speed railway cutting scenario," in Electron. Lett., vol. 47, no. 21, pp. 1167-1168, Oct. 2011.

[71] R. He, Z. Zhong, B. Ai, J. Ding, and Y. Yang, "Propagation measurements and analysis of fading behavior for high speed rail cutting scenarios," in Proc. IEEE GLOBALCOM'12, California, USA, Dec. 2012, pp. 5015-5020.

[72] R. He, Z. Zhong, B. Ai, J. Ding, Y. Yang, and A. F. Molisch, "Short-term fading behavior in high-speed railway cutting scenario: measurements, analysis, and statistical models," IEEE Trans. Antennas Propag., vol. 61, no. 4, pp. 2209-2222, Apr. 2013.

[73] R. Sun, C. Tao, L. Liu, and Z. Tan, "Channel measurement and characterization for HSR U-Shape groove scenarios at $2.35 \mathrm{GHz}$," in Proc. IEEE VTC'13-Fall, Las Vegas, USA, Sept. 2013, pp. 1-5.

[74] L. Tian, J. Zhang, and C. Pan, "Small scale fading characteristics of wideband radio channel in the U-Shape cutting of high-speed railway," in Proc. IEEE VTC'13-Fall, Las Vegas, USA, Sept. 2013, pp. 1-6.

[75] F. Luan, Y. Zhang, L. Xiao, C. Zhou, and S. Zhou, "Fading characteristics of wireless channel on high-speed railway in hilly terrain scenario," Int. J. Antennas Propag., vol. 2013, Article ID 378407, 9 pages, 2013. doi: $10.1155 / 2013 / 378407$.

[76] Wang Qian; Xu Chunxiu; Wu Muqing; Zhao Min; Yu Deshui, "Propagation characteristics of high speed railway radio channel based on broadband measurements at $2.6 \mathrm{GHz}$,' in Proc. IEEE WCNC'14, Istanbul, Turkey, Apr. 2014, pp. 166-170.

[77] Y. Zhang, Z. He, W. Zhang, L. Xiao, and S. Zhou, "Measurementbased delay and Doppler characterizations for high-speed railway hilly scenario," Int. J. Antennas and Propag., vol. 2014, Article ID 875345, 8 pages, 2014. doi:10.1155/2014/875345.

[78] P. Aikio, R. Gruber, and P. Vainikainen, "Wideband radio channel measurements for train tunnels," in Proc. IEEE VTC'98, Ottawa, Canada, May 1998, pp. 460-464.

[79] C. Briso-Rodriguez, J. M. Cruz, and J. I. Alonso, "Measurements and modeling of distributed antenna systems in railway tunnels," IEEE Trans. Veh. Technol., vol. 56, no. 5, pp. 2870-2879, Sept. 2007.

[80] T. Zhou, C. Tao, L. Liu, and Z. Tan, "Ricean K-Factor measurements and analysis for wideband radio channels in high-speed railway U-Shape cutting scenarios,' in Proc. IEEE VTC'14-Spring, Seoul, Korea, May 2014, pp. 1-5. 
[81] K. Guan, Z. Zhong, B. Ai, and T. Kürner, "Propagation measurements and analysis for train stations of high-speed railway at $930 \mathrm{MHz}$," IEEE Trans. Veh. Technol., vol. 63, no. 8, pp. 3349-3516, Oct. 2014.

[82] K. Guan, Z. Zhong, B. Ai, and T. Kurner, "Empirical models for extra propagation loss of train stations on high-speed railway," IEEE Trans. Antennas Propag., vol. 62, no. 3, pp. 1395-1408, Mar. 2014.

[83] J. Lu, G. Zhu, and B. Ai, "Fading analysis for the high speed railway viaduct and terrain cutting scenarios," Int. J. Antennas and Propag., vol. 2012, Article ID 862945, 9 pages, 2012.

[84] K. Guan, Z. Zhong, B. Ai, and T. Kürner, "Propagation measurements and modeling of crossing bridges on high-speed railway at $930 \mathrm{MHz}$," IEEE Trans. Veh. Technol., vol. 63, no. 8, pp. 502-517, Feb. 2014.

[85] Y. Wen, Y. Ma, X. Zhang, X. Jin, and F. Wang, "Channel fading statistics in high-speed mobile environment," in Proc. IEEE APWC'12, Cape Town, South Africa, Sept. 2012, pp. 1209-1212.

[86] B. Chen, Z. Zhong, and B. Ai, "Stationarity intervals of time-variant channel in high speed railway scenario," J. China Commun., vol. 9, no. 8, pp. 64-70, Aug. 2012.

[87] R. He, D. Zhong, B. Ai, and C. Oestges, "Shadow fading correlation in high-speed railway environments," IEEE Trans. Veh. Technol., vol. 64, no. 7, pp. 2762-2772, July 2015.

[88] R. He, Z. Zhong, B. Ai, and B. Zhang, "Measurement-based autocorrelation model of shadow fading for the high-speed railways in urban, suburban, and rural environments," in Proc. APSURSI'14, Memphis, TN, USA, July 2014, pp. 949-950.

[89] J. Qiu, C. Tao, L. Liu, and Z. Tan, "Broadband channel measurement for the high-speed railway based on WCDMA," in Proc. IEEE VTC'12Spring, Yokohama, Japan, May 2012, pp. 1-5.

[90] L. Liu, C. Tao, T. Zhou, Y. Zhao, X. Yin, and H. Chen, "A highly efficient channel sounding method based on cellular communications for high-speed railway scenarios," EURASIP J. Wireless Commun. Networking, vol. 2012, 16 pages, 2012. doi:10.1186/1687-1499-2012-307.

[91] X. Yin, X. Cai, X. Cheng, J. Chen, and M. Tian, "Empirical geometrybased random-cluster model for high-speed-train channels in UMTS networks," IEEE Trans. Intell. Transp. Syst., vol. 16, no. 5, pp. 28502861, Oct. 2015.

[92] L. Tian and J. Zhang, "Analysis on non-stationary characteristics of wideband radio channel in HSR U-shape cutting scenario," in Proc. IEEE ICCC'14, Shanghai, China, Oct. 2014, pp. 544-548.

[93] K. Guan, Z. D. Zhong, J. I. Alonso, and C. BrisoRodriguez,"Measurement of distributed antenna system at $2.4 \mathrm{GHz}$ in a realistic subway tunnel environment," IEEE Trans. Veh. Technol., vol. 61, no. 2, pp. 834-837, Feb. 2012.

[94] K. Guan, Z. D. Zhong, and B. Ai,"Statistic modeling for propagation in tunnels based on distributed antenna systems," in Proc. IEEE APSURSI'13, Florida, USA, July 2013, pp. 1920-1921.

[95] L. Tian, X. Yin, Q. Zuo, J. Zhou, Z. Zhong, and S. Lu, "Channel modeling based on random propagation graphs for high speed railway scenarios," in Proc. IEEE PIMRC'12, Sydney, Australia, Sept. 2012, pp. $1746-1750$.

[96] S. Knörzer, M. A. Baldauf, T. Fugen, and W. Wiesbeck, "Channel analysis for an OFDM-MISO train communications system using different antennas," in Proc. IEEE VTC'07-Fall, Baltimore, USA, Oct. 2007, pp. 809-813.

[97] K. Guan, Z. Zhong, B. Ai, and T. Kürner, "Deterministic propagation modeling for the realistic high-speed railway environment," in Proc. IEEE VTC'13-Spring, Dresden, Germany, June 2013, pp. 1-5.

[98] D. J. Cichon, T. C. Becker, and W. Wiesbeck, "Determination of timevariant radio links in high-speed train tunnels by ray optical modeling," in Proc. IEEE APS/URSI'95, California, USA, June 1995, pp. 508-511.

[99] D. J. Cichon, T. Zwick, and W. Wiesbeck, "Ray optical modeling of wireless communications in high-speed railway tunnels," in Proc. IEEE VTC'96-Spring, Atlanta, USA, May 1996, pp. 546-550.

[100] K. Guan, Z. Zhong, B. Ai, and T. Kurner, "Semi-deterministic pathloss modeling for viaduct and cutting scenarios of high-speed railway," IEEE Antennas Wireless Propag. Lett, vol. 12, pp. 789-792, June 2013.

[101] B. Chen and Z. Zhong, "Geometry-based stochastic modeling for MIMO channel in high-speed mobile scenario," Int. J. Antennas and Propagation, vol. 2012, Article ID 184682, 6 pages, 2012. doi: $10.1155 / 2012 / 184682$

[102] Q. Zheng, C. Xu, and M. Wu, "A novel MIMO channel model for high speed railway system," in Proc. IEEE ICCT'12, Chengdu, China, Nov. 2012, pp. 31-35.

[103] A. Ghazal, C.-X. Wang, H. Haas, M. A. Beach, X. Lu, and D. Yuan, "A non-stationary MIMO channel model for high speed train communication systems," in Proc. IEEE VTC'12-Spring, Yokohama, Japan, May 2012, pp. 1-5.
[104] A. Ghazal, C.-X. Wang, H. Haas, M. Beach, R. Mesleh, D. Yuan, X. Ge, and M. K. Chahine, "A non-stationary geometry-based stochastic model for MIMO high-speed train channels," invited paper, in Proc. ITST'12, Taipei, Taiwan, Nov. 2012, pp. 7-11.

[105] A. Ghazal, C.-X. Wang, B. Ai, D. Yuan, and H. Haas, "A nonstationary wideband MIMO channel model for high-mobility intelligent transportation systems," IEEE Trans. Intell. Transp. Syst., vol. 16, no. 2, pp. 885-897, Apr. 2015.

[106] A. Ghazal, C.-X. Wang, Y. Liu, P. Fan, and M. K. Chahine, "A generic non-stationary MIMO channel model for different high-speed train scenarios," in Proc. IEEE ICCC'15, Shenzhen, China, Nov. 2015.

[107] M. Patzold, Mobile Radio Channels. 2nd ed. Chichester: Wiley, 2011.

[108] B. Chen, Z. Zhong, B. Ai, and D. Michelson, "A geometry-based stochastic channel model for high-speed railway cutting scenarios," IEEE Antennas Wireless Propag. Lett., vol. 14, pp. 851-854, Apr. 2015.

[109] S. Lin, Z. Zhong, L. Cai, and Y Luo, "Finite state Markov modelling for high speed railway wireless communication channel," in Proc. IEEE GLOBECOM'12, California, USA, Dec. 2012, pp. 5421-5426.

[110] S. Lin, L. Kong, L. He, K. Guan, B. Ai, Z. Zhong, and C. Rodriguez, "Finite-state Markov modeling for high-speed railway fading channels," IEEE Antennas Wireless Propag. Lett., vol. 14, pp. 954-957, Apr. 2015.

[111] L. Liu, C. Tao, J. Qiu, T. Zhou, R. Sun, and H. Chen, "The dynamic evolution of multipath components in high-speed railway in viaduct scenarios: From the birth-death process point of view," in Proc. IEEE PIMRC'12, Sydney, Australia, Sept. 2012, pp. 1774-1778.

[112] L. Xuan, S. Chao, B. Ai, and Z. Gang "Finite-state Markov modeling of fading channels: A field measurement in high-speed railways," in Proc. IEEE ICCC'13, Xi'an, China, Aug. 2013, pp. 577-582.

[113] Y. Liu, C.-X. Wang, A. Ghazal, S. Wu, and W. Zhang, "A multimode waveguide tunnel channel model for high-speed train wireless communication systems", in Proc. EuCAP'15, Lisbon, Portugal, Apr. 2015.

[114] R. He, Z. Zhong, and C. Briso, "Broadband channel long delay cluster measurements and analysis at $2.4 \mathrm{GHz}$ in subway tunnels," in Proc. IEEE VTC'11-Spring, Budapest, Hungary, May 2011, pp. 1-5.

[115] R. He, B. Ai, Z. Zhong, A. F. Molisch, R. Chen, and Y. Yang, "A measurement-based stochastic model for high-speed railway channels," IEEE Trans. Intell. Transp. Syst., vol. 16, no. 3, pp. 1120-1135, June 2015.

[116] 3GPP TR 36.873 V12.2.0, "Study on 3D channel model for LTE," June 2015.

[117] B. Mondal, T. Thomas, E. Visotsky, F. Vook, A. Ghosh, Y.-H. Nam, Y. Li, J. Zhang, M. Zhang, Q. Luo, Y. Kakishima, K. Kitao, "3D channel model in 3GPP," IEEE Commun. Mag., vol. 53, no. 3, pp. 16-23, Mar. 2015.

[118] M. Shafi, M. Zhang, P. J. Smith, A. L. Moustakas, and A. F. Molisch, "The impact of elevation angle on MIMO capacity," in Proc. IEEE ICC'06, Istanbul, Turkey, June 2006, pp. 4155-4160.

[119] J. Meinila, P. Kyosti, L. Hentila, T. Jamsa, E. Suikkanen, E. Kunnari, and M. Narandzia, "D5.3: WINNER+ final channel models," CELTIC/CP5-026, June 2010.

[120] T. A. Thomas, F. W. Vook, E. Mellios, G. S. Hilton, A. R. Nix, and E. Visotsky, "3D Extension of the 3GPP/ITU Channel Model," in Proc. IEEE VTC'13-Spring, Dresden, Germany, June 2013, pp. 1-5.

[121] R. Almesaeed, A. S. Ameen, E. Mellios, A. Doufexi, and A. R. Nix, "A proposed 3D extension to the 3GPP/ITU channel model for $800 \mathrm{MHz}$ and $2.6 \mathrm{GHz}$ bands," in Proc. EuCAP'14, Hague, Netherlands, Apr. 2014, pp. 3039-3043.

[122] P. Liu, B. Ai, Z. Zhong, and X. Zhou, A novel train-to-train communication model design based on multihop in high-speed railway, Int. J. Antennas and Propag., vol. 2012, Article ID 475492, 9 pages, 2012. doi: $10.1155 / 2012 / 475492$

[123] P. Liu, X. Zhou, and Z. Zhong, "Outage analysis of train-to-train communication model over Nakagami- $m$ channel in high-speed railway," Int. J. Antennas and Propag., vol. 2013, Article ID 617895, 10 pages, 2013. doi: $10.1155 / 2013 / 617895$

[124] Q. Luo, W. Fang, T. Yang, and D. Wang, "A transmit beamforming algorithm for high-speed train communication," in Proc. IEEE VTC'12Fall, Québec City, Canada, Sept. 2012, pp. 1-5.

[125] H.-H. Wang and H.-A. Hou, "Experimental analysis of beamforming in high-speed railway communication," in Proc. IEEE PIMRC'11, Toronto, Canada, Sept. 2011, pp. 745-749.

[126] H. Zhu, "Radio resource allocation for OFDMA systems in high speed environments," IEEE J. Sel. Areas Commun., vol. 30, no. 4, pp. 748-759, May 2012. 
[127] M. Cheng, X. Fang, and L. Yan, "Beamforming and Alamouti STBC combined downlink transmission schemes in communication systems for high-speed railway," in Proc. WCSP'13, Hangzhou, China, Oct. 2013, pp. $1-6$.

[128] J. Yao, S. S. Kanhere, and M. Hassan, "Mobile broadband performance measured from high-speed regional trains," in Proc. IEEE VTC'11-Fall, San Francisco, USA, Sept. 2011, pp. 1-5.

[129] Y. Dong, P. Fan, and K. Letaief, "High speed railway wireless communications: efficiency v.s. fairness," IEEE Trans. Veh. Technol., vol. 63, no. 2, pp. 925-930, Feb. 2014.

[130] B. Chen, Z. Zhong, B. Ai, and X. Chen, "Comparison of antenna arrays for MIMO system in high speed mobile scenarios," in Proc. IEEE VTC'11-Spring, Budapest, Hungary, May 2011, pp. 1-5.

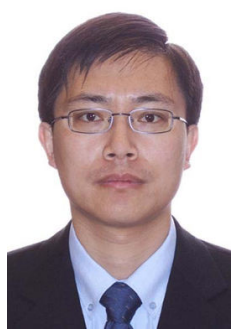

Cheng-Xiang Wang (S'01-M'05-SM'08) received the BSc and MEng degrees in Communication and Information Systems from Shandong University, China, in 1997 and 2000, respectively, and the $\mathrm{PhD}$ degree in Wireless Communications from Aalborg University, Denmark, in 2004.

He has been with Heriot-Watt University, Edinburgh, U.K., since 2005, and was promoted to a Professor in wireless communications in 2011. He is also an Honorary Fellow of the University of Edinburgh, U.K., and a Chair/Guest Professor of Shandong University and Southeast University, China. He was a Research Fellow at the University of Agder, Grimstad, Norway, from 2001-2005, a Visiting Researcher at Siemens AG-Mobile Phones, Munich, Germany, in 2004, and a Research Assistant at Technical University of Hamburg-Harburg, Hamburg, Germany, from 2000-2001. His current research interests focus on wireless channel modelling and $5 \mathrm{G}$ wireless communication networks, including green communications, cognitive radio networks, high mobility communication networks, massive MIMO, millimeter wave communications, and visible light communications. He has edited 1 book and published over 220 papers in refereed journals and conference proceedings.

Prof. Wang served or is currently serving as an editor for 8 international journals, including IEEE Transactions on Vehicular Technology (since 2011) and IEEE Transactions on Wireless Communications (2007-2009). He was the leading Guest Editor for IEEE Journal on Selected Areas in Communications, Special Issue on Vehicular Communications and Networks. He served or is serving as a TPC member, TPC Chair, and General Chair for over 80 international conferences. He received the Best Paper Awards from IEEE Globecom 2010, IEEE ICCT 2011, ITST 2012, IEEE VTC 2013-Spring, and IWCMC 2015. He is a Fellow of the IET, a Fellow of the HEA, and a member of EPSRC Peer Review College.

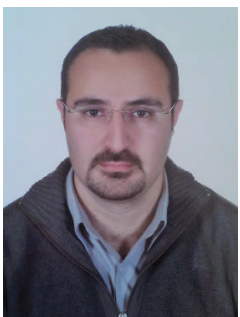

Ammar Ghazal (S'15) received the BSc degree in Electronics and Telecommunication Engineering from Damascus University, Syria in 2006, MSc by Research and $\mathrm{PhD}$ from Heriot-Watt University, Edinburgh, U.K., in 2010 and 2015, respectively. His main research interests include wireless propagation channel characterization and modeling, non-stationary channel models, and high-speed train wireless propagation.

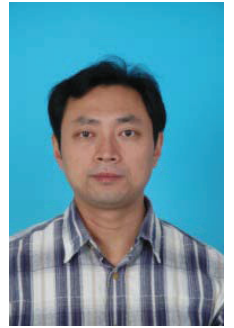

Bo Ai (M'00-SM'09) received his Master and Ph.D. degrees from Xidian University in 2002 and 2004 in China, respectively. He graduated in 2007 with great honors of Excellent Postdoctoral Research Fellow in Tsinghua University. He is now working in Beijing Jiaotong University as a professor and advisor of $\mathrm{Ph}$. D. candidates. He is a deputy director of State Key Laboratory of Rail Traffic Control and Safety. He is an associate editor for IEEE Trans. on Consumer Electronics and an editorial committee member of journal of Wireless Personal Communications. He has authored/co-authored 5 books, 26 invention patents, and 220 scientific research papers in his research area till now. His current research interests include broadband and dedicated mobile communication theories and technologies for GSM-R and LTE-R, with emphasis on channel measurements and modeling. He is a Fellow of the IET.

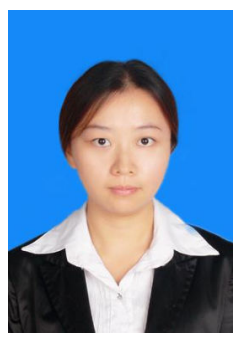

Yu Liu received the BSc and MEng degrees in communication and information systems from Qufu Normal University, China, in 2010 and 2013, respectively. Since September 2013, she has been a $\mathrm{PhD}$ student in the School of Information Science and Engineering (ISE) at Shandong University, China. Her main research interests include non-stationary channel modeling, high-speed train wireless propagation characterization and modeling, and channel modeling for special scenarios.

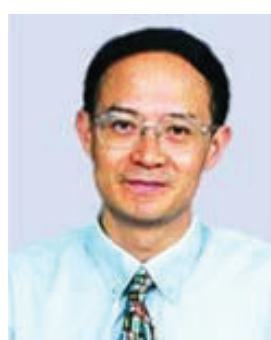

Pingzhi Fan (M'93-SM'99-F'15) received his $\mathrm{PhD}$ degree in Electronic Engineering from the Hull University, UK.

He is currently a Professor and Director of the Institute of Mobile Communications, Southwest Jiaotong University, China. He is a recipient of the UK ORS Award, the Outstanding Young Scientist Award by NSFC, and the chief scientist of a national 973 research project. He served as general chair or TPC chair of a number of international conferences, and is the guest editor-in-chief, guest editor or editorial member of several international journals. He is the founding chair of IEEE VTS BJ Chapter and ComSoc CD Chapter, the founding chair of IEEE Chengdu Section. He also served as a board member of IEEE Region 10, IET(IEE) Council and IET Asia-Pacific Region. He has over 200 research papers published in various academic English journals (IEEE/IEE/IEICE, etc.), and 8 books (incl. edited), and is the inventor of 20 granted patents. His research interests include high mobility wireless communications, 5G technologies, wireless networks for big data, signal design \& coding, etc. 